Revista de Matemática: Teoría y Aplicaciones 2003 10(1-2) : 23-36

CIMPA - UCR - CCSS ISSN: 1409-2433

\title{
METODOLOGÍA ESTADÍSTICA: BASES PARA EL ESTABLECIMIENTO DE POLÍTICAS ADECUADAS PARA EL MANEJO DEL ARBOLADO URBANO EN LA CIUDAD DE MÉXICO.
}

\author{
Alejandro Aldama* Héctor J. VÁzquez ${ }^{\dagger}$ Jaime Grabinsky ${ }^{\ddagger}$ \\ Alicia Chacalo
}

Recibido (versión revisada): 16 Jun 2003

\begin{abstract}
Resumen
Con el fin de evaluar la calidad del bosque urbano un análisis estadístico exploratorio usando un enfoque arborí stico y un enfoque ecológico de una muestra de árboles de las calles de la Ciudad de México fue seguido de un estudio confirmatorio usando an álisis de senderos para probar hipótesis sobre relaciones entre diferentes variables. Todo esto con el fin de evaluar y administrar la calidad del arbolado de las calles de la Ciudad de México.
\end{abstract}

Palabras clave: análisis factorial, componentes principales, análisis de correspondencias, análisis de conglomerados, medidas de asociación, análisis de senderos, arbolado urbano.

\begin{abstract}
An Exploratory analysis using arboricultural and ecological statistical approaches of a representative sample of Mexico City's street trees were followed by a confirmatory study through Path Analysis to test hypothesis stating causal relationships among different variables. All this in order to evaluate and to manage the quality of Mexico City's Urban Forest.
\end{abstract}

\footnotetext{
${ }^{*}$ Departamento de Sistemas, Universidad Autónoma Metropolitana, Unidad Azcapotzalco, Av. San Pablo 180, Col. Reynosa Tamaulipas, 02200, México D.F., México. Tel. +(52)(55) 53189094 , Fax 53 9445 34. E-Mail: alao@correo.azc.uam.mx

${ }^{\dagger}$ Departamento de Sistemas, misma dirección que A. Aldama. E-Mail: hjv@correo.azc.uam.mx

${ }^{\ddagger}$ Departamento de Ciencias Básicas, misma dirección que A. Aldama. E-Mail: jags@correo.azc.uam.mx

${ }^{\S}$ Departamento de Energía, misma dirección que A. Aldama. E-Mail: ach@correo.azc.uam.mx
} 
Keywords: factor analysis, principal components, correspondence analysis, cluster analysis, measures of association, path analysis, urban trees.

Mathematics Subject Classification: 62H20, 62H25, 62H30, 62H99

\section{Introducción.}

La segunda mitad del siglo veinte se caracterizó por el acelerado desarrollo económico y tecnológico, y la consecuente concentració n en áreas urbanas. Entre 1950 y 2000 la población en la República Mexicana aumentó de 28.5 millones (14.8 hab/km2) a más de 100 millones de habitantes $(52.2 \mathrm{hab} / \mathrm{km} 2)$. El impacto de tal incremento ha tenido efectos globales negativos sobre la naturaleza y la calidad de vida [5]. En particular el arbolado en un sistema urbano juega un papel preponderante para controlar los niveles de contaminación atmosf érica. Asimismo, forma un elemento esencial en la arquitectura del paisaje y en el buen funcionamiento de microclimas urbanos.

Recomendaciones para un adecuado desarrollo de áreas verdes en sistemas urbanos han sido propuestas por varias organizaciones internacionales tales como las Naciones Unidas y el Banco Interamericano de Desarrollo [10], [11]. Inclusive se han desarrollado programas de apoyo económico para realizar campañas de plantación y mantenimiento.

La ciudad de México se ha convertido en la más poblada del mundo. Durante las últimas décadas se han invertido recursos económicos considerables en programas extensivos de plantación de árboles. Sin embargo dichos programas no han considerado características de los sitios de plantación; han carecido de criterios para mantener una densidad y una diversidad adecuadas; tampoco han integrado programas de sensibilización para el cuidado y buen manejo de los árboles. Es por esto que resulta necesaria una evaluación global y sistemática sobre la situación del arbolado, y así poder diseñar y planear campañas adecuadas de plantación y mantenimiento.

El objetivo principal de este estudio es presentar los principios de una metodología estadística para estudiar los bosques urbanos, con la intención de obtener una estimación de las características individuales de cada árbol; evaluar relaciones entre diferentes individuos de la población y entre diferentes factores, tomados aisladamente y en su conjunto. Todo esto con el fin de establecer prácticas adecuadas para el buen desarrollo del arbolado.

\section{Metodología y Discusión}

Se considera que la problemática del arbolado urbano es de carácter eco-sistémico. Mirenowicz y Garnier [9] proponen integrar diferentes punto de vista. El arquitectónico del paisaje, el sociológico, el descriptivo y el ecológico. La Figura 1 describe el eco-sistema de arbolado. Se incluyen: 1.-factores controlables, 2.-respuestas esperadas clasificadas en dos grupos $(2 \mathrm{a}, 2 \mathrm{~b}), 3$--perturbaciones o factores no controlables y 4.-posibles interacciones entre estas. Este esquema es típico de la metodología experimental, no obstante, en el presente estudio observacional, permite orientar la estrategia hacia la comprensión de las posibles relaciones entre las variables involucradas. 
Figura 1: Ecosistema de Arbolado Urbano.

Para este estudio, se efectuó un muestreo representativo en el Distrito Federal. Dado que los límites de la Ciudad de México no están definidos con precisión, se consideró como dominio de estudio el Distrito Federal, conformado por 16 delegaciones. La muestra consistió de 1260 árboles distribuidos de manera proporcional al tamaño de cada delegación. Se consideraron solamente árboles localizados sobre las banquetas, quedando excluidos aquellos plantados en las jardineras viales (camellones), parques públicos o reservas ecológicas. Para cada árbol se reconocieron 67 variables (primarias y secundarias), entre las cuales destacan, las características del árbol (especie, diámetro, altura, etc.), la localización y la evaluación de sitio, la condición general de salud, las enfermedades, presencia de insectos, heridas, tratamientos recomendados y nivel socioeconómico de cada una de las 227 manzanas estudiadas. El levantamiento de la información y el trabajo de campo fue realizado por 17 estudiantes de ingeniería ambiental, como parte de su formación en Administración y Manejo de Arbolado Urbano.

Inicialmente se obtuvieron las estadísticas descriptivas básicas para todo el Distrito Federal, por Delegación, por Manzana y por Especie [2]. Las más significativas son: especies dominantes, distribución de diámetros a la altura del pecho, distribución de estaturas, problemas de salud, evaluación del sitio y tratamientos requeridos. El $41 \%$ de los árboles pertenecen a las especies que superan el 5\% ( Fresno, Troeno y Cedro) de la muestra, denominadas, en arboricultura, dominantes y el $49 \%$ se encuentran en sitios buenos o muy buenos. El $39 \%$ tienen una altura de a lo más $3 \mathrm{~m}$ y sólo el $15 \%$ tienen diámetros superiores a $48 \mathrm{~cm}$. Alrededor del $50 \%$ se encuentran en buenas condiciones de salud

Posteriormente se evaluaron las asociaciones y correlaciones entre variables con diferentes unidades estadísticas (manzana, delegación, especie). Se entiende por manzana al mínimo espacio urbano destinado para habitación o actividad económica, delimitado por vialidades. El Distrito Federal esta dividido en 16 circunscripciones políticas llamadas delegaciones. Como resultados relevantes se mencionan: grandes diferencias entre las delegaciones y entre manzanas en cuanto al número de árboles; asociación significativa entre 
Figura 2: Modelos asociados a la distribución espacial de individuos.

la calidad del sitio de plantación y la condición de los árboles; falta de relación directa entre el nivel socioeconómico y la condición de los árboles; asociación entre el diámetro del árbol y la especie; reducido número de especies diferentes para ciertas delegaciones, distribuciones de densidad de árboles por manzana y por delegación inadecuadas [3].

También se estudió la muestra desde el punto vista ecológico (ver Figura 1, variables $2 \mathrm{~b}$ ). Los ecosistemas son fundamentalmente estructurados en el espacio y en el tiempo. Con base en esto se han propuesto diferentes índices. En este estudio solo se presenta el cálculo de tres de ellos: Distribución espacial, Diversidad y Asociación inter-especies, Ludwig \& Reynolds (1988). Se consideraron únicamente las especies dominantes (Cedro, Olmo, Casuarina, Fresno, Eucalipto, Jacaranda, Colorín). El uso de estos índices en ecosistemas urbanos ha generado controversias. Sin embargo algunos autores han señalado aspectos de interés para la cuantificación de la estructura de ecosistemas urbanos [1].

\subsection{Distribución Espacial}

Estos índices están definidos mediante la razón varianza/media, la cuál evalúa el grado de agrupación o agregación de individuos en un espacio discreto. Los tres tipos de patrones de acuerdo a esta razón y los modelos probabilísticos correspondientes se presentan en la Figura 2, así como los valores de los índices de dispersión (ID) y de Green (IG).

Para las especies dominantes se observan valores de ID mayores a 1 e IG's entre 0 y 1 (Tabla 1), lo que sugiere que los individuos se agrupan en cúmulos.

\subsection{Diversidad}

Este índice está constituido por dos componentes, la abundancia (número de especies) y la equidad o regularidad. Frontier [6] ejemplifica estos conceptos así: "en 100 especies, si una o dos constituyen la casi totalidad del efectivo y las restantes son raras, se obtendrá una diversidad débil. Si por el contrario, las especies están representadas por frecuencias similares, se observa una diversidad elevada. Este segundo componente es la regularidad ". 


\begin{tabular}{c|cc}
\hline \hline Especie & ID & IG \\
\hline Cedro & 6.82 & 0.046 \\
Eucalipto & 2.19 & 0.016 \\
Olmo & 3.97 & 0.043 \\
Troeno & 5.94 & 0.030 \\
Casuarina & 5.77 & 0.072 \\
Jacaranda & 5.27 & 0.043 \\
Fresno & 6.77 & 0.026 \\
Colorín & 4.27 & 0.450 \\
\hline \hline
\end{tabular}

Tabla 1: Valores de índices de dispersión para especies dominantes.

Se observa que las delegaciones con mayor diversidad son Tlalpan, Iztapalapa, Gustavo A. Madero y Miguel Hidalgo. No obstante, las delegaciones con menor diversidad no se identificaron dado que algunos casos arrojaron valores contradictorios [13].

En arboricultura una alternativa para definir la Diversidad es como el número de especies dominantes en una muestra. Algunos resultados se presentan en [3].

\subsection{Asociación Inter-especies}

Para evaluar la asociación entre especies se usa una tabla binaria en donde se señalan la presencia o ausencia de especies por delegación. Es posible evaluar la asociación entre pares de especies o entre conjuntos mayores. En el caso de las ocho especies dominantes se obtienen así 28 combinaciones. Para cada par se obtienen los índices de Jaccard, Ochiai y Dice [8]. Estos índices son consistentes y permiten establecer una jerarquía de asociación de dos en dos. La evaluación de la asociación de las ocho especies dominantes en conjunto se hizo por medio del estadístico $\chi^{2}$ en el intervalo bilateral del $90 \%$ de confianza y no se observó asociación, a nivel delegación, entre las especies dominantes.

Cada uno de estos tres enfoques (descriptivo univariado, bivariado y ecológico) derivaron conclusiones sobre aspectos específicos, relacionados con las frecuencias de valores, factores, modalidades o aspectos en cuanto a la estructura ecológica de la muestra. Sin embargo, no fue posible obtener una visión integral de las variables y sus posibles interrelaciones.

Para obtener una visión integral y encontrar un conjunto de variables más parsimonioso, se aplicaron tres técnicas del Análisis Multivariado: Análisis de Correspondencias Múltiples (ACM), Análisis en Componentes Principales (ACP), y Análisis de Conglomerados Jerárquico (ACJ) [7]. En los análisis se consideraron las siguientes variables: Especie, Nivel Socioeconómico, Distribución de Edades, Densidad de Árboles por Manzana, Condición General, Evaluación del Sitio y Diversidad de Especies.

El Análisis de Correspondencias Múltiple, aplicado al árbol como unidad de estudio, mostró una asociación entre los diferentes niveles de los índices de Evaluación de Sitio y Condición General. La Condición General se derivó de las variables relacionadas con enfermedades, estructura del árbol, heridas e insectos. En tanto que Evaluación de Sitio, fue conformada por el nivel de compactación del suelo y las interferencias a los árboles. 
Esto apoya la idea básica de que la elección y cuidado del sitio de plantación es fundamental para la supervivencia y buena condición del árbol.

Debido a que al considerar el árbol como unidad de estudio, no se redujo la varianza, se consideraron la Delegación, la Manzana y la Especie, como unidades de estudio.

\subsection{Análisis por Delegación}

Las variables consideradas fueron: Condición General, Evaluación del Sitio, Nivel Socioeconómico, Densidad de Árboles por Manzana, Diversidad de Especies y Distribución de Edades.

En el caso de Condición General, Evaluación del Sitio, Nivel Socioeconómico se consideraron solamente las cuatro categorías más altas. La Densidad de árboles por manzana se estimó mediante un conteo rápido sobre una muestra de 866 manzanas. Para la Diversidad de Especies se consideró el 80\% de los árboles en cada delegación. La Distribución de Edades se estimó mediante la distribución del diámetro [12]. Valores pequeños corresponden a distribuciones de edades cercanas a la ideal propuesta por Richards. Para comparar la distribución ideal y la distribución observada de diámetros (o edades), se utilizó la métrica $\chi^{2}[7]$.

Del ACP aplicado a las delegaciones se obtuvo que más del $80 \%$ de la varianza se explica mediante tres ejes (Figura 3). La Condición General y la Evaluación del Sitio se asocian con el primer eje (33.5\% del total de la varianza), al igual que la Densidad de Árboles por Manzana y el Nivel Socioeconómico. La Diversidad de Especies por Delegación y la Distribución de Edades se asocia con el segundo eje (31.6\% de la varianza total). El tercer eje $(18.2 \%)$ resultó asociado con la Distribución de Edades y Diversidad de Especies.

El primer eje fue tomado como «la calidad de adecuación al sitio» de los árboles sobre sitios específicos, en tanto que el segundo eje se consideró como un indicador de la diversidad biológica y temporal. Las cinco delegaciones: Magdalena Contreras, Xochimilco, Iztacalco, Gustavo A. Madero y Milpa Alta, muestran una buena calidad de adecuación al sitio; lo opuesto se observó en las delegaciones: Alvaro Obregón, Iztapalapa, Benito Juárez y Cuauhtémoc. Mientras que Milpa Alta, Gustavo A Madero, Iztapalapa y Alvaro Obregón mostraron tener una diversidad de especies elevada. De la Figura 3 se puede inferir que en tanto más alto sea el nivel Socioeconómico, la densidad de árboles es mayor. Las delegaciones Benito Juárez y Cuauhtémoc mostraron los valores más elevados.

\begin{tabular}{|l|l|l|l|l|l|l|}
\hline & $\mathrm{X} 1$ & $\mathrm{X} 2$ & $\mathrm{X} 3$ & $\mathrm{X} 4$ & $\mathrm{X} 5$ & $\mathrm{X} 6$ \\
\hline X1=Diversidad de Especies & 1.00 & & & & & \\
\hline X2=Condición General & -0.08 & 1.00 & & & & \\
\hline X3=Evaluación del Sitio & -0.12 & $\mathbf{0 . 7 4}$ & 1.00 & & & \\
\hline X4=Nivel Socioeconómico & -0.14 & -0.10 & -0.06 & 1.00 & & \\
\hline $\begin{array}{c}\text { X5=Densidad de Árboles } \\
\text { por Manzana }\end{array}$ & -0.22 & -0.42 & -0.01 & $\mathbf{0 . 6 2}$ & 1.00 & \\
\hline X6=Distribución de Edades & $\mathbf{0 . 5 4}$ & -0.25 & -0.04 & -0.36 & 0.07 & 1.00 \\
\hline
\end{tabular}

Tabla 2. Matriz de correlaciones entre las variables de la delegación 
Figura 3: Proyección de delegaciones y variables sobre los dos primeros ejes resultado del Análisis en Componentes Principales.

La matriz de correlaciones (Tabla 2) confirma algunas relaciones por pares de variables. Las más altas son entre los pares:

- Condición General y Evaluación del Sitio

- Nivel Socioeconómico y Densidad de Árboles por Manzana

- Diversidad de Especies y Distribución de Edades.

Los conglomerados para delegaciones (Figura 4) sugieren una asociación geográfica y quizás histórica: periféricas o centrales, recientes o antiguas, montañosas o ribereñas (en los alrededores de antiguos lagos y parques), así como, densa o escasamente pobladas.

Estos resultados sugieren la formulación de las siguientes hipótesis:

1. Delegaciones con los mejores sitios corresponden a delegaciones con árboles en mejor condición.

2. Delegaciones pertenecientes a los niveles socioeconómicos más altos tienen una mayor densidad de árboles por manzana.

3. Una mejor distribución de edades corresponde a una mayor diversidad de especies.

Por primera vez el nivel socio económico mostró un efecto benéfico en el arbolado urbano, mostrando una correlación con la densidad.

La correlación entre la Distribución de Edades y la Diversidad de Especies por delegación, podría indicar una mayor longevidad. Esto podría explicar la recomendación propuesta por Richards en cuanto a que un incremento en la diversidad aumenta la estabilidad [12]. 
Figura 4: Dendrogramas resultado de un Análisis de Conglomerados Jerárquico para Delegaciones y sus variables.

Para confirmar las hipótesis hasta aquí planteadas se utilizó el método de análisis de senderos, de acuerdo a la metodología propuesta por Bentler-Weeks en [4]. Estudios previos han generado las hipótesis representadas esquemáticamente en la Figura 5.

Mediante el programa EQS Structured Equations Program [4] se genera la solución estándar:

$$
\begin{gathered}
\text { DiversidaddeEspecies }=+0.562 . \text { Distribución de Edades }+0.066 . \text { Nivel } \\
\text { Socioeconómico }+0.841 . \text { E } 1 \\
\text { CondiciónGeneral }=-.028 . \text { DiversidaddeEspecies }- \\
\text { 0.541.DensidaddeArbolespor Manzana }-0.057 . \text { DistribucióndeEdades }+ \\
\text { 781.EvaluacióndelSitio }+0.260 . \text { NivelSocioeconómico }+0.441 . E 2 \\
\text { DensidaddeArbolespor Manzana }=0.618 . \text { NivelSocioeconómico }+0.786 . E 5 \\
\text { DistribucióndeEdades }= \\
\text {-0.059.EvaluacióndelSitio }-0.364 . \text { NivelSocioeconómico }+0.930 . \text { E6 }
\end{gathered}
$$

Los resultados muestran una distribución de los residuos estandarizados cercana a la distribución ideal (simétrica y centrada en cero). Comparando el valor $\chi^{2}$ (15 grados de libertad) suponiendo independencia completa entre las variables con el valor de $\chi^{2}(6$ grados de libertad), se confirma la significancia del modelo (p-value $<0.01$ ). El índice de comparación de ajuste resultó 0.939 confirmando un buen ajuste. El cumplimiento de estos criterios es suficiente para validar las hipótesis propuestas [4].

La solución estándar final es: 
Figura 5: Diagrama de senderos correspondiente a las hipótesis establecidas para características de los árboles en las delegaciones.

Figura 6: Diagrama de senderos, resultado de las pruebas de significancí a de los parámetros del modelo anterior (cf. Figura 5). 


\section{DiversidaddeEspecies $=0.537$. DistribucióndeEdades $+0.843 . E 1$; \\ CondiciónGeneral $=$ -0.415.EvaluacióndeSitio + 0.733.DensidaddeArbolespor Manzana + 0.538.E2; \\ DensidaddeArbolespor Manzana $=+0.618$. NivelSocioeconómico + 0.786.E5;}

Sin embargo, solamente las relaciones mostradas en la Figura 6 resultaron significativas (cf. hipótesis 1, 2, y 3). Para este caso las pruebas de independencia $\chi^{2}$ para las variables y para el modelo también resultaron significativas $(\mathrm{p}<0.01)$. El índice de comparación de ajuste resultó igual a 0.939 . Sin embargo el estudio de los residuos entre cada variable revela falta de ajuste entre Distribución de Edades y Nivel Socioeconómico y entre Distribución de Edades y Condición General. Lo que muestra que la modelación no se logra con un solo criterio.

\subsection{Análisis por Especies}

Un estudio análogo se llevo a cabo para las 8 especies más abundantes de la muestra, cada una caracterizada por cuatro variables: Condición General, Evaluación de Sitio, Nivel Socioeconómico y Distribución de Edades. Del ACP se obtuvo $80 \%$ de la varianza en dos ejes. El primer eje muestra oposición entre la Evaluación del Sitio y la Distribución de Edades. El segundo se relaciona más con el Nivel Socioeconómico. El ACP permitió obtener tres grupos de especies:

- Pino, Eucalipto y Colorín, con mejor Distribución de Edades

- Troeno, Jacaranda y Fresno, con buen Nivel Socioeconómico

- Cedro y Olmo, con mejor Condición General y mejor Evaluación del Sitio.

Del análisis de senderos no se obtuvo un modelo válido, posiblemente por el tamaño pequeño de la muestra.

\subsection{Análisis por Manzana.}

Las variables consideradas para caracterizar la manzana como unidad estadística son: Condición General, Evaluación del Sitio, Nivel Socioeconómico, Distribución de Edades y Diversidad de Especies.

Del ACP resultó que tres ejes incluyen el $80 \%$ de la varianza. La Condición General y la Evaluación del Sitio se asocian al primer eje (Figura 7). La Diversidad y la Distribución de Edades se asocian con el segundo. El Nivel Socioeconómico se asocia con el tercer eje (Figura 8).

El primer eje puede considerarse como una medida de la calidad de adaptación del árbol al sitio, mientras que el segundo eje relaciona la diversidad de especies y la distribución de edades. El tercer eje permite clasificar las manzanas de acuerdo a buenos y malos niveles 
Figura 7: Proyecciones de las manzanas y variables en los dos primeros ejes resultado del ACP.

Figura 8: Proyecciones de las manzanas y sus variables sobre los ejes 2 y 3 resultados. 
Figura 9: Dendrogramas resultado del Análisis de Conglomerados Jerárquico para las variables de manzanas.

socioeconómicos. La eliminación de la distribución de edades y del nivel socioeconómico, permite distinguir seis grupos de manzanas (Figura 7).

Del Análisis de Conglomerados resultaron tres grupos de variables (Figura 9):

- Evaluación del Sitio, Condición General

- Nivel Socioeconómico

- Diversidad de Especies, Distribución de Edades.

La Tabla 3 de correlaciones muestra asociaciones débiles entre variables.

\begin{tabular}{|l|l|l|l|l|l|}
\hline & $Y 1$ & $Y 2$ & $Y 3$ & $Y 4$ & $Y 5$ \\
\hline$Y 1=$ Condición General & 1.00 & & & & \\
\hline$Y 2=$ Evaluación del Sitio & $\mathbf{0 . 5 5}$ & 1.00 & & & \\
\hline$Y 3=$ Nivel Socioeconómico & 0.00 & 0.12 & 1.00 & & \\
\hline$Y 4=$ Distribución de Edades & -0.01 & 0.12 & 0.10 & 1.00 & \\
\hline$Y 5=$ Diversidad de Especies & 0.00 & 0.10 & -0.03 & $\mathbf{0 . 3 2}$ & 1.00 \\
\hline
\end{tabular}

Tabla 3. Matriz de correlaciones entre variables de manzanas

No obstante, del análisis de senderos resultó el siguiente modelo:

CondiciónGeneral $=+0.567 *$ EvaluacióndelSitio +0.820 E 1

DensidaddeArboles $=+0.323 *$ DistribucióndeEdades $+0.942 E 5$

Información global sobre la adecuación de ajuste se presenta en la Tabla 4. 


\begin{tabular}{|l|l|}
\hline Modelo de Independencia $\chi^{2}$ (10g.l.) & 1122.66 \\
\hline modelo (3 g.l.) & 8.457 \\
\hline valor p de la estadística $\chi^{2}$ & 0.03774 \\
\hline Índice de Bentler-Bonett & 0.931 \\
\hline Índice Comparativo de Ajuste (CFI) & 0.952 \\
\hline
\end{tabular}

Table 4. Resultados de EQS.

Estos resultados proporcionan una distribución de residuos cercana a la distribución ideal (simétrica y centrada en cero) y apoyan la significancia del modelo.

\section{Conclusiones y Recomendaciones.}

Los aspectos que podrían resaltarse son:

- No se pudo obtener una reducción de variables para toda la muestra en su conjunto.

- Los resultados de las pruebas estadísticas permitieron establecer comparaciones entre delegaciones, manzanas y especies dominantes mediante el uso de indicadores robustos.

- Las variables fueron clasificadas en grupos y se obtuvieron interpretaciones para agrupaciones de variables tales como: calidad ecológica, esto es, la evaluación conjunta de la calidad entre el sitio y el árbol.

- La diversidad de especies por delegación y distribución de edades resultaron altamente asociadas. Esto sugiere que una mayor diversificación de especies proporciona una mayor supervivencia para los árboles. Dado que la distribución de edades fue estimada solamente con base en la longitud del diámetro, este resultado requiere un aná lisis más detallado.

- Varios de los resultados obtenidos indican que el potencial de salud del bosque esta lejos de ser alcanzado. La mayor parte de los individuos son árboles jóvenes; a una parte substancial de los individuos maduros se le ha aplicado podas inadecuadas, no cuentan con espacios suficientes y propicios para su crecimiento, y presentan heridas en sus hojas y troncos. Por ser en su mayoría jóvenes no proporcionan buena sombra y no representan un elemento estético para la arquitectura del paisaje. Tampoco protegen contra la contaminación, y por tanto producen un efecto global de salud muy precario.

- Las ecuaciones resultantes del análisis confirmatorio de senderos sugieren el estudio detallado de otros aspectos arborísticos. No parece que resultados como los presentados aquí hayan sido publicados en la literatura, y por tanto se sugiere que se realicen más investigaciones en la misma dirección. Con base en esto se podrían proponer políticas tendientes al mejoramiento de la calidad del arbolado urbano. 
El uso sistemático de inventarios, el conocimiento de los factores controlables y de sus relaciones con las características deseadas de un bosque pueden permitir, mediante estudios estadísticos integrales, comprender y establecer mecanismos para el mejor desarrollo de un bosque urbano.

\section{Referencias}

[1] Berdoulay (1999) "L'écologie urbaine : un récit contre nature?". En: Ville et Environnement, de l'Écologie Urbaine á la Ville Durable. Barnier, V. \& Tucolet, C. (Eds.), La Documentation Française, Num. 89.

[2] Chacalo, A.; Aldama, A.; Grabinsky, J. (1994) "Street tree inventory in Mexico City", Journal of Arboriculture 20(4): 222-226.

[3] Chacalo, A.; Grabinsky, J.; Aldama, A. (1996) "Inventario del arbolado de alineación de la Ciudad de México", Ciencia Forestal 21(79), México.

[4] Dunn, G.; Everitt, B.; Pickels, A. (1993) Modelling Covariances and Latent Variables Using EQS. Chapman \& Hall, London.

[5] Emangard, P. H. (1999) "Du caractère non durable de certaines décisions d'urbanisme", en: : Ville et Environnement, De l'Écologie Urbaine á la Ville Durable. V. Barnier \& C. Tucolet, (Eds.), La Documentation Française, Num. 89.

[6] Frontier, S. (1999) Les Ecosystèmes. Presses Universitaires de France. Paris, France.

[7] Lebart, L.; Morineau, A.; Piron, M. (1995) Statistique Exploratoire Multidimensionnelle. Dunod, France.

[8] Ludwig, J. A.; Reynolds, J. F. (1988) Statistical Ecology. John Wiley \& Sons, New York.

[9] Mirenowicz, P.; Garnier, C. (1999) "La ville et l'écologie, entre discours et pratique, annés 1980: un appel pour penser la ville autrement", en: Ville et Environnement, De l'Écologie Urbaine á la Ville Durable. V. Barnier \& C. Tucolet, (Eds), La Documentation Française, Num. 89.

[10] Nilsson, K.; Randrup, T.; Tvedt, T. (1996) "Technological aspects of urban greening", Draft presented at the International Seminar on Urban Greening in Latin America and the Caribbean. Mexico City, BID, Gobierno del Estado de México, México.

[11] Sorensen, M., (1996) "Introducción a la Creación de las Áreas Verdes Urbanas", No.ENV 96-103. BID, 71pp. Washington, D. C.

[12] Richards, N. A. (1983) "Diversity and stability in a street tree population", Urban Ecology 7: $159-171$.

[13] Vázquez, H. J.; Grabinsky, J.; Chacalo, A.; Aldama, A. (1998) "Estudio exploratorio de índices ecológicos en una muestra de arbolado urbano de la Ciudad de México", en: Memorias del XIII Foro Nacional de Estadística, Asociación Mexicana de Estadística, Monterrey, México: 191-199. 